\title{
Symmetry breaking in monoatomic 2-lattices
}

\author{
Giuseppe Fadda and Giovanni Zanzotto \\ Dipartimento di Metodi e Modelli Matematici per le Scienze Applicate - DMMMSA \\ Università di Padova, Via Belzoni 7, 35131 Padova, Italy \\ e-mail: zanzotto@dmsa.unipd.it
}

\begin{abstract}
In this paper we describe all the possibilities for symmetry breaking transformations in monoatomic 2-lattices (crystal structures with two identical points in their unit translational cell). This is done by establishing the symmetry hierarchies (partial ordering) for the arithmetic classes of symmetry groups of these crystals (Fig. 1). We also study the 'Ericksen-Pitteri neighbourhoods' for them, thus making a local analysis of their configuration space. We give details about two physically relevant cases, analysing the neighbourhoods and the possible symmetry-breaking mechanisms for the diamond and the hexagonal close-packed structures (Figs. 2 and 3).
\end{abstract}

\section{Introduction}

A general theory for the investigation of the arithmetic symmetry of crystalline lattices has been proposed in [25, 26], based on earlier work [6, 22, 24] on crystal symmetry and the invariance of their constitutive equations. This approach, summarised in $\S \S 2-3$, has been applied in $[14,15]$ to obtain a systematic classification of the distinct arithmetic crystalline types in the two simplest cases, that is, for the two- and three-dimensional lattices with two identical atoms in their unit cells (called respectively the 'monoatomic 2nets' and 'monoatomic 2-lattices'1).

In this paper we continue the investigation of monoatomic 2-lattices begun in [15], giving all their symmetry hierarchies, and describing, as a consequence, all the ways in which these structures can undergo symmetrybreaking transformations. ${ }^{2}$

\footnotetext{
${ }^{1}$ Many examples of such lattices are found in nature - see for instance at the end of $\S 5.2$ and Table 1.

${ }^{2}$ Here we consider the (small but finite) lattice distortions that cause 'direct' symmetry losses in lattices, which are the case in most phase transformations in crystalline materials; our analysis, however, also allows for the study of symmetry loss arising from large deformations. We do not consider transformations involving discontinuities in the crystal periodicity, such as in phase transitions with cell-doubling and analogous phenomena, which involve the special lattice descriptors excluded in formulas (7)-(8).
} 
Our main result is summarised in Fig. 1, which shows the symmetry hierarchies existing for the 29 arithmetic types of monoatomic 2-lattices found in [15] (the latter are described in Tables 1 and 2). Such hierarchies derive from the partial ordering for the arithmetic conjugacy classes of the symmetry groups of monoatomic 2-lattices, discussed in $\S \S 3-4$. Our analysis extends to monoatomic 2-lattices the results on simple lattices (or '1-lattices') that are classical in crystallography - see for instance [5, 21, 26].

We also investigate in detail the local structure of the configuration space of monoatomic 2-lattices (see the definitions in formulas (3)-(5) below), studying their 'Ericksen-Pitteri (E-P) neighbourhoods' - see [7, 8, 23, 24, 18, $26]$. The latter are important in the modelling of phase transitions in crystals because they are the natural domains for the constitutive functions of crystalline materials that undergo symmetry-breaking transformations (see also $[2,19,26])$.

We give an explicit description of the E-P neighbourhoods in two wellknown and physically important examples, that is, for the hexagonal closepacked 'h.c.p.' structure (this monoatomic 2-lattice gives one example of densest lattice packing of equal spheres in three dimensions), and for the diamond structure.

The neighbourhoods of the diamond structure are relevant for the kinematics and energetics of the well-known $\alpha-\beta$ transition in Sn ('tin pest', briefly discussed in $\S 6.1$ ), and possibly for other materials, such as $\mathrm{C}$ and $\mathrm{Si}$, that also crystallise in this fashion. The neighbourhoods of the h.c.p. structure are relevant for the phase transitions of the various 'hexagonal metals' that assume this densest-packing atomic arrangement (these neighbourhoods also arise in the theory of twinning in $\alpha$-U, see [12]). The examples we consider actually illustrate how to analyse the E-P neighbourhoods for any crystal structure $([29,26]$ treat the prototypical case of simple lattices). See $[3,9,10,11,13,1,26]$ for recent work on the symmetry and constitutive equations of complex crystals undergoing solid-state transformations.

Acknowledgements. This work has been performed within the TMR Network FMRX-CT 98-0229 of the E.U. G.Z. also acknowledges the partial funding of the Italian Cofinanziamento MURST 98 'Modelli matematici per la scienza dei materiali'.

\section{Three-dimensional 2-lattices}

We recall some basic notions discussed in $[25,26,15]$, where further details can be found. 


\section{$2.1 \quad$ 2-lattices}

A 2-lattice is an infinite and discrete subset $\mathcal{M}$ of points in the affine space $\mathbb{A}^{3}$, coinciding with the union of two 'affine simple lattices' (1-lattices):

$$
\mathcal{M}=\mathcal{M}\left(\mathbf{e}_{a}, \mathbf{p}\right)=\left\{\mathrm{O}+\mathcal{L}\left(\mathbf{e}_{a}\right)\right\} \bigcup\left\{\mathrm{O}+\mathbf{p}+\mathcal{L}\left(\mathbf{e}_{a}\right)\right\} .
$$

Here $\mathrm{O}$ is the origin of $\mathbb{A}^{3}, \mathcal{L}\left(\mathbf{e}_{a}\right)$ denotes a (linear) simple lattice in the translation space $\mathbb{R}^{3}$ of $\mathbb{A}^{3}$, generated by the basis $\mathbf{e}_{a},(a=1,2,3)$ :

$$
\mathcal{L}\left(\mathbf{e}_{a}\right)=\left\{\mathbf{v} \in \mathbb{R}^{3}: \mathbf{v}=v^{a} \mathbf{e}_{a}, v^{a} \in \mathbb{Z}\right\}
$$

$\mathcal{L}\left(\mathbf{e}_{a}\right)$ is called the skeletal lattice of $\mathcal{M}$.

The vector $\mathbf{p}=p^{a} \mathbf{e}_{a}, a=1,2,3$ in (1) is called the 'shift' of $\mathcal{M}$ : it gives the separation of the two simple lattices constituting $\mathcal{M}$. The vectors $\left(\mathbf{e}_{1}, \mathbf{e}_{2}, \mathbf{e}_{3}, \mathbf{p}\right)$ are called the descriptors of $\mathcal{M}$; they must satisfy the conditions:

$$
\mathbf{e}_{1} \cdot \mathbf{e}_{2} \times \mathbf{e}_{3} \neq 0 \quad \text { and } \quad \mathbf{p} \neq l^{a} \mathbf{e}_{a}, \quad \text { for } l^{a} \in \mathbb{Z}, a=1,2,3 .
$$

Where convenient, we denote the above vectors by $\boldsymbol{\varepsilon}_{\sigma}, \sigma=1,2,3,4$ :

$$
\varepsilon_{a}=\mathbf{e}_{a}, \quad a=1,2,3, \quad \text { and } \quad \varepsilon_{4}=\mathbf{p} .
$$

In this paper we only consider monoatomic 2-lattices, whose points are all physically indistinguishable.

The set of all 4-tuples of vectors of $\mathbb{R}^{4}$ satisfying conditions (3) is denoted by $\mathcal{D}_{3,1}$ and is called the space of descriptors, or the configuration space of monoatomic 2-lattices. Let $\mathcal{Q}_{4}$ denote the ten-dimensional vector space of all symmetric 4 by 4 real matrices; in $\mathcal{Q}_{4}$ consider the nine-dimensional (nonlinear) submanifold $\mathcal{Q}_{3,1}$ collecting all the 2-lattice metrics $K$, defined by:

$$
K=\left(K_{\sigma \tau}\right), \quad K_{\tau \sigma}=K_{\sigma \tau}=\varepsilon_{\sigma} \cdot \varepsilon_{\tau} \quad \text { for } \quad \varepsilon_{\sigma} \in \mathcal{D}_{3,1}
$$

$(\sigma, \tau=1,2,3,4)$, where the $\varepsilon_{\sigma}$ satisfy conditions (3)-(4). Some properties of the space $\mathcal{Q}_{3,1}$ are given in [15].

The 2-lattice metric $K$ is independent of the orientation of a multilattice in $\mathbb{A}^{3}$, and owing to the Galilean invariance of the constitutive functions, also $\mathcal{Q}_{3,1}$ is referred to as the 'configuration space' of 2-lattices.

We notice that certain nonessential descriptors $\varepsilon_{\sigma}$ of monoatomic 2lattices actually give a 1-lattice in $\mathbb{A}^{3}$ (see for instance $[25,26]$ ). Explicitly, in the configuration space $\mathcal{D}_{3,1}$ the nonessential descriptors are those for which $\mathbf{p}=\frac{1}{2} \beta^{a} \mathbf{e}_{a}+\mathbf{t}$, where $\mathbf{t} \in \mathcal{L}\left(\mathbf{e}_{a}\right)$ and the numbers $\beta^{a}$ are

either $(1,1,1)$ or a permutation of $(1,1,0)$ or $(1,0,0)$. 
We confine ourselves to the portions of the configuration spaces of monoatomic 2-lattices that only contain essential descriptors, which generate monoatomic 2-lattices that are not 1-lattices. Explicitly, we consider

$$
\mathcal{D}_{3,1}^{e s s}=\left\{\left(\mathbf{e}_{a}, \mathbf{p}\right) \in \mathcal{D}_{3,1}: \mathbf{p} \neq \frac{1}{2} \beta^{a} \mathbf{e}_{a}+\mathbf{t}, \beta^{a} \text { as in }(6)\right\} \subset \mathcal{D}_{3,1},
$$

and

$$
\mathcal{Q}_{3,1}^{\text {ess }}=\left\{K \in \mathcal{Q}_{3,1}: K_{\sigma \tau}=\varepsilon_{\sigma} \cdot \varepsilon_{\tau}, \varepsilon_{\sigma} \in \mathcal{D}_{3,1}^{\text {ess }}\right\} \subset \mathcal{Q}_{3,1} .
$$

For brevity, also the spaces $\mathcal{D}_{3,1}^{e s s}$ and $\mathcal{Q}_{3,1}^{\text {ess }}$ are referred to as the 'configuration spaces' of 2-lattices.

\section{The symmetry groups of monoatomic 2-lattices; their action on the configuration spaces}

As discussed in [25, 26] (see also Proposition 1 in [15]), the indeterminacy in the choice of the essential descriptors of a given 2-lattice (up to overall translations) leads to consider the following discrete group of 'global symmetry' $($ see $[24]):^{3}$

$$
\Gamma_{3,1}<G L(4, \mathbb{Z}),
$$

constituted by the unimodular integral 4 by 4 matrices which, by definition, have the following structure: for $a, b=1,2,3$,

$$
\mu \in \Gamma_{3,1} \quad \Leftrightarrow \quad \mu_{\sigma}^{\tau}=\left(\begin{array}{ccc|c} 
& & & l^{1} \\
& m_{a}^{b} & & l^{2} \\
& & & l^{3} \\
\hline 0 & 0 & 0 & \alpha
\end{array}\right),
$$

where $\left(m_{a}^{b}\right)$ is any matrix in $G L(3, \mathbb{Z}), l^{b} \in \mathbb{Z}$, and $\alpha= \pm 1$. The group $\Gamma_{3,1}$ defines an action on $\mathcal{D}_{3,1}^{\text {ess }}$ :

$$
\bar{\varepsilon}_{\sigma}=\mu_{\sigma}^{\tau} \varepsilon_{\tau}, \quad \mu \in \Gamma_{3,1}
$$

this in turn induces the following action on the space $\mathcal{Q}_{3,1}^{\text {ess }}$ of (essential) 2-lattice metrics:

$$
K \mapsto \bar{K}=\mu^{T} K \mu
$$

Based on these actions, the arithmetic symmetry of monoatomic 2-lattices is studied as the natural generalisation of the classical procedure used in crystallography for 1-lattices; the group $\Gamma_{3,1}$ defined above plays for 2-lattices

\footnotetext{
${ }^{3} G L(n, \mathbb{Z})$ denotes the group of all the $\mathrm{n}$ by $\mathrm{n}$ invertible matrices with integral entries.
} 
the same role played for 1-lattices by the arithmetic group $G L(3, \mathbb{Z})$ - see for instance [5, 27, 21, 26].

The arithmetic classification of 2-lattice symmetry is based on the analysis of the subgroups of $\Gamma_{3,1}$ that act isometrically on some 2-lattice (or, equivalently, that stabilise some 2-lattice metric under the action (12)), and that are maximal for this property.

For any $\varepsilon_{\sigma} \in \mathcal{D}_{3,1}^{\text {ess }}$ with metric $K \in \mathcal{Q}_{3,1}^{\text {ess }}$, we define the lattice group $\Lambda\left(\varepsilon_{\sigma}\right)$ :

$$
\Lambda\left(\varepsilon_{\sigma}\right)<\Gamma_{3,1}<G L(4, \mathbb{Z}),
$$

as the subgroup of all the integral matrices $\mu \in \Gamma_{3,1}$ such that

$$
\Lambda\left(\varepsilon_{\sigma}\right)=\left\{\mu \in \Gamma_{3,1}: \mu^{T} K \mu=K\right\}=\Lambda(K) .
$$

As is immediatly checked, the lattice group is necessarily finite and is independent of the orientation of the 2-lattice in $\mathbb{A}^{3}$.

By (14), under a change of descriptors $\overline{\boldsymbol{\varepsilon}}_{\sigma}=\mu_{\sigma}^{\tau} \varepsilon_{\tau}$ for the 2-lattice $\mathcal{M}\left(\varepsilon_{\sigma}\right)$ as in (11), the lattice group transforms as follows:

$$
\Lambda\left(\mu_{\sigma}^{\tau} \varepsilon_{\tau}\right)=\mu^{-1} \Lambda\left(\varepsilon_{\sigma}\right) \mu \text { for all } \mu \in \Gamma_{3,1} .
$$

Formula (15) along with Proposition 1 in [15] say that any given 2-lattice

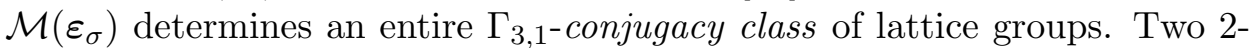
lattices $\mathcal{M}$ and $\mathcal{M}^{\prime}$ are defined to be of the same arithmetic type when their lattice groups are $\Gamma_{3,1}$-conjugate. We also say that two metrics $K$ and $K^{\prime}$ (or two sets of descriptors $\varepsilon_{\sigma}$ and $\varepsilon_{\sigma}^{\prime}$ ) are of the same arithmetic type when their lattice groups are conjugate in $\Gamma_{3,1}$. This generates a subdivision of $\mathcal{Q}_{3,1}^{\text {ess }}$ into equivalence classes (the 'strata' of the action (12)), which are called the arithmetic types within $\mathcal{Q}_{3,1}^{\text {ess }}$ (analogously for $\mathcal{D}_{3,1}^{\text {ess }}$, when the orthogonal transformations are also taken into account).

In [15] we have determined all the distinct conjugacy classes of lattice groups in $\Gamma_{3,1}$, and described the resulting 29 arithmetic symmetry types' of monoatomic 2-lattices in three dimensions. ${ }^{4}$ These types are listed in Table $1 ;{ }^{5}$ Table 2 gives a sketch of their conventional cells.

One main way of obtaining information about the action of $\Gamma_{3,1}$ on the configuration space $\mathcal{Q}_{3,1}^{\text {ess }}$ is studying the sets of metrics that share the same

\footnotetext{
${ }^{4}$ Similarly, one obtains the classical 14 Bravais types in three dimensions by considering the conjugacy classes of lattice groups ('arithmetic holohedries') within $G L(3, \mathbb{Z})$, which for 1-lattices plays the role that is played by the group $\Gamma_{3,1}$ for monoatomic 2-lattices.

${ }^{5}$ We recall (see $[25,26]$ ) that the finite group $\Lambda\left(\varepsilon_{\sigma}\right)$ carries more information than the mere point group of $\mathcal{M}\left(\varepsilon_{\sigma}\right)$ : indeed, unlike with the latter, given the lattic group $\Lambda\left(\varepsilon_{\sigma}\right)$ it is possible to reconstruct uniquely the (isomorphism class of the) space group of $\mathcal{M}\left(\varepsilon_{\sigma}\right)$ see Proposition 5 in [25]. Furthermore, the arithmetic classification of multilattices is in general finer than their space-group classification. For instance, it can be seen in Table 1 that some arithmetic types share the same space group (like the hexagonal types 26 and 28 ), although they do not have arithmetically equivalent lattice groups.
} 
lattice group. Thus, given any subgroup $\Lambda$ of $\Gamma_{3,1}$, we define the fixed set $I(\Lambda) \subset \mathcal{Q}_{3,1}^{\text {ess }}$ of $\Lambda$ as follows:

$$
I(\Lambda)=\left\{K \in \mathcal{Q}_{3,1}^{e s s}: \mu^{T} K \mu=K \text { for all } \mu \in \Lambda\right\}
$$

(hereafter we shall always consider $\Lambda$ to be a lattice group as in (14), unless otherwise stated). It can be seen that, by its very definition, if considered in the vector space $\mathcal{Q}_{4}$ rather than in $\mathcal{Q}_{3,1}^{\text {ess }}$, the fixed set $I(\Lambda)$ is a linear subspace which contains as subspaces of strictly smaller dimension the fixed sets of any larger lattice group:

$$
I\left(\Lambda^{\prime}\right) \subset I(\Lambda) \quad \Leftrightarrow \quad \Lambda<\Lambda^{\prime} .
$$

These inclusion relations for the fixed sets are very important in the theory of phase transitions, as they give the symmetry hierarchies and the kinematic possibilities for symmetry breaking in crystal structures. This is precisely what is investigated in this paper in the case of monoatomic 2-lattices.

It is not difficult to check that the lattice groups in the same $\Gamma_{3,1^{-}}$ conjugacy class as in (15) have fixed sets that are related through the action (12):

$$
I\left(\mu^{-1} \Lambda \mu\right)=\mu^{T} I(\Lambda) \mu, \quad \mu \in \Gamma_{3,1} .
$$

This means that each one of the 29 arithmetic types in $\mathcal{Q}_{3,1}^{\text {ess }}$ is given by an 'orbit' of fixed sets as in (18). Table 1 lists the skeletal metrics and shift vectors, and hence the equations of a representative fixed set, for each arithmetic type in $\mathcal{Q}_{3,1}^{\text {ess }}$.

\section{The 29 arithmetic types of monoatomic 2-lattices and their symmetry hierarchies}

\subsection{The 29 types (Tables 1 and 2)}

In [15] we have described the 29 distinct arithmetic types of monoatomic 2-lattices. They originate from the existence of 29 distinct conjugacy classes of lattice groups in $\Gamma_{3,1}$. The non-unit generators of a representative lattice group for each such class are given in Table 1 (because all monoatomic 2lattices are centro-symmetric, -1 is also a generator for the lattice groups in $\Gamma_{3,1}$, which for brevity is not indicated). Table 2 shows the cells of the 2-lattices corresponding to each class.

Notice that given a monoatomic 2-lattice type, a unique arithmetic class in $G L(3, \mathbb{Z})$ remains defined; in turn, the latter determines a unique Bravais type of 1-lattices, which we call the the Bravais type of the given 2-lattice type (roughly, this corresponds to the skeletal type of the 2-lattice ${ }^{6}$ ). Tables

\footnotetext{
${ }^{6}$ However, see the comments made in $[20,14,15]$ about 'excess skeletal symmetry' in multilattices.
} 
1 and 2 list the 2-lattice types grouping them according to their Bravais type. Notice that for each of the latter, there are in general several distinct monoatomic 2-lattice types, depending on how many 'essentially distinct ways' there exist of placing the extra point in the unit Bravais cell. ${ }^{7}$

We remark that several, but not all, of the 29 monoatomic 2-lattice types give crystal structures of materials existing in nature: ${ }^{8}$ type number 14 corresponds to the so-called 'A20 structures' ${ }^{\text {' }}$ (such as $\alpha$-uranium); type 22 corresponds to the 'A5 structures' (for instance, $\beta$-tin); type 23 gives the so-called 'bct5 structure' recently proposed for Si (body-centred tetragonal silicon with five-fold coordination, see [4]); type 24 corresponds to the 'A7 structures' (as in bismuth); number 27, for a special ratio of the hexagonal lattice parameters, gives the h.c.p. structure ('A6'), found in nature in many metals (for generic values of that ratio, which are still compatible with type 27 , it gives a 'distorted' h.c.p. structure, which is also often found in nature); finally the only cubic type, number 29 , corresponds to the 'diamond structure A4'. ${ }^{10}$

Notice that type number 25 has hexagonal Bravais type but trigonal space group lacking a six-fold symmetry operation: this is the only monoatomic 2-lattice type whose crystal class is not holohedral, as all of the other types have holohedral point and lattice groups.

\subsection{Symmetry hierarchies: partial ordering of the conjugacy classes of lattice groups in $\Gamma_{3,1}$ (Figure 1)}

Eight of the 29 lattice groups listed in Table 1, that is, groups number $10,20,21,23,26,27,28$ and 29 , are maximal in $\Gamma_{3,1}$ : they are not contained in any larger lattice group (this property is obviously preserved under $\Gamma_{3,1}$-conjugacy); the corresponding 2-lattice types have therefore maximal symmetry. Among them are, necessarily, the three holohedral hexagonal types 26-28, and the holohedral face-centred cubic type 29; however, unlike with 1-lattices, some 2-lattices with maximal symmetry have Bravais types that are not maximally symmetric: this is what happens with the primitive and centred tetragonal types 20,21, 23 and the primitive orthorhombic type 10.

Up to $\Gamma_{3,1}$-conjugacy, all the other lattice groups in Table 1 are subgroups of at least one of the maximal lattice groups indicated above. These group-

\footnotetext{
${ }^{7}$ Notice that, for the centred Bravais types, in Table 2 we represent the non-unit conventional cells, whose several points are connected by suitable 'centring vectors'.

${ }^{8}$ It is not surprising that we do not find all the 29 types of monoatomic 2-lattices in nature; the same is true also for the 14 Bravais types of 1-lattices, only a few of which are actually observed, so far, as crystal structures of actual materials.

${ }^{9}$ The symbols like A20, etc., refer to the 'Strukturberichte' [28], a widely used empirical catalog of crystal structures.

${ }^{10}$ Neither primitive nor body-centered cubic Bravais types are possible for monoatomic 2-lattices.
} 
subgroup relations give a partial ordering for the 29 conjugacy classes of lattice groups, which is represented in the diagram of Fig. 1. This is the main result of this paper, as it gives the symmetry hierarchies for the 29 monoatomic 2-lattices types (notice that the minimal lattice group is the triclinic group $\{1, \overline{1}\}$, which is contained in all of the other groups, because, as noted earlier, all monoatomic 2-lattices are centro-symmetric). We omit the proof of these inclusion relations, which is somewhat tedious and can be established in several equivalent ways.

Our Fig. 1 is but the generalisation, to monoatomic 2-lattices, of Fig. 4.2 in [21], which shows the symmetry hierarchies of the classical 14 Bravais types of 1-lattices (that is, the partial ordering for the 14 conjugacy classes of lattice groups - 'Bravais arithmetic classes' - that exist in $G L(3, \mathbb{Z})$ ). As each one of the 29 2-lattice types determines a unique Bravais type, one can check that the partial ordering of the latter is reflected in that of the former: see the hierarchies of the 'boxes' in Fig. 1, which represent the Bravais types of the 2-lattices (as mentioned earlier, two Bravais types are missing from the diagram in that figure).

The information contained in Fig. 1 is relevant to the modelling of phase transitions in crystals because it shows in how many different ways monoatomic 2-lattices can undergo 'direct' symmetry-breaking process. When a conjugacy class (or symmetry type) is 'higher' than another one in the partial ordering of Fig. 1, it means that a 2-lattice of the first type can be directly deformed (that is, with a small distortion) into a 2-lattice of the second type. A comparison of Fig. 1 with the cell representations in Table 2 illustrates this: some of the lattice deformations are obvious, other are geometrically less transparent.

We will see in the next Sections how the partial ordering of Fig. 1 can be related to the local structure of the configuration spaces $\mathcal{Q}_{3,1}^{\text {ess }}$ or $\mathcal{D}_{3,1}^{\text {ess }}$ of monoatomic 2-lattices. This link gives complete information about the symmetry-breaking mechanisms that exist for such crystal structures. We summarise some related notions and definitions in the next Section, giving details about two physically interesting examples in the two final Sections (see also [29, 26] for 1-lattices).

\section{Ericksen-Pitteri neighbourhoods}

\subsection{The neighbourhoods}

In order to describe the local structure of the configuration spaces $\mathcal{Q}_{3,1}^{\text {ess }}$ (or $\left.\mathcal{D}_{3,1}^{\text {ess }}\right)$ and to determine the details of the symmetry-breaking mechanisms in monoatomic 2-lattices, we first recall a result showing that the action (12) of the infinite group $\Gamma_{3,1}$ on $\mathcal{Q}_{3,1}^{\text {ess }}$ reduces, in suitably restricted domains, to the action of the lattice groups (hence of finite groups). 
Proposition 5.1 For any metric $K$ in $\mathcal{Q}_{3,1}^{\text {ess }}$, there exists a neighbourhood $\mathcal{N}(K)$ of $K$ in $\mathcal{Q}_{3,1}^{\text {ess }}$ such that

(i) $\mu^{T} \mathcal{N}(K) \mu=\mathcal{N}(K) \quad \forall \mu \in \Lambda(K) \quad$ (that is, $\left.\forall \mu: \mu^{T} K \mu=K\right)$;

(ii) $\mu^{T} \mathcal{N}(K) \mu \cap \mathcal{N}(K)=\emptyset \quad \forall \mu \in \Gamma_{3,1} \backslash \Lambda(K)$.

See $[7,8,23,24]$ for more details and a proof of this Proposition. A set $\mathcal{N}(K)$ with the properties above is called an 'Ericksen-Pitteri (E-P) neighbourhood' of the centre $K$.

As a consequence of the above Proposition, we have that, near $K$, 'symmetry cannot increase':

Proposition 5.2 Given any $K \in \mathcal{Q}_{3,1}^{\text {ess }}$,

$$
\forall K^{\prime} \in \mathcal{N}(K), \quad \Lambda\left(K^{\prime}\right) \leq \Lambda(K)
$$

As the metrics in $\mathcal{N}(K)$ have at most symmetry $\Lambda(K)$, one needs to study the inclusion relations for the subgroups of $\Lambda(K)$ that are themselves lattice groups, in order to find which direct symmetry-breaking transitions are possible for a crystal with metric $K$ (see [20] for similar conclusions regarding the space groups, but recall the remarks in footnote 5 ).

\subsection{Variants; structure of the neighbourhoods}

When a phase transition occurs, with a crystal passing from a configuration with metric $K$ to one nearby with metric $K_{1}$ not too far from $K$ (that is, $K_{1}$ belonging to some E-P neighbourhood $\mathcal{N}(K)$ ), the crystal is as likely to access also the configurations whose metrics are in the $\Lambda(K)$-orbit of $K_{1}$. We call such orbit, which is all in $\mathcal{N}(K)$, a variant structure $\mathcal{V}\left(K_{1}\right)$ in $\mathcal{N}(K)$ :

$$
\forall K_{1} \in \mathcal{N}(K), \quad \mathcal{V}\left(K_{1}\right)=\left\{\mu^{T} K_{1} \mu: \mu \in \Lambda(K)\right\} .
$$

The elements of $\mathcal{V}\left(K_{1}\right)$ are called the $K$-variants of $K_{1}$, or simply, its variants; by definition, all the variants of $K_{1}$ have the same arithmetic type. The variants are very important in the theory of twinning and microstructure formation (see the literature quoted in the Introduction).

The number of variants and the number of their distinct lattice groups (which are all conjugate subgroups of $\Lambda(K)$ ) are given by the following Proposition:

Proposition 5.3 The lattice groups of the $K$-variants of a metric $K_{1}$ are conjugate subgroups of the lattice group $\Lambda(K)$ :

$$
\forall K_{1} \in \mathcal{N}(K), \forall \mu \in \Lambda(K), \quad \Lambda\left(\mu^{T} K_{1} \mu\right)=\mu^{-1} \Lambda\left(K_{1}\right) \mu .
$$

The number $n$ of variants is the index of $\Lambda\left(K_{1}\right)$ in $\Lambda(K)$, and the number $m$ of distinct lattice groups (and thus of fixed sets) corresponding to $\mathcal{V}\left(K_{1}\right)$ is the index of the normaliser of $\Lambda\left(K_{1}\right)$ in $\Lambda(K)$, with $1 \leq m \leq n$. 
The Propositions above give us a way now to describe the local structure of the space $\mathcal{Q}_{3,1}^{e s s}$ near any metric $K$, that is, the structure of an E-P neighbourhood $\mathcal{N}(K)$. We have seen before that symmetry in $\mathcal{N}(K)$ is dictated by the lattice group $\Lambda(K)$, that in $\mathcal{N}(K)$ we can only find metrics that are invariant under subgroups of $\Lambda(K)$, and that the variants in $\mathcal{N}(K)$ are grouped into structures whose lattice groups are all $\Lambda(K)$-conjugate.

Therefore it is important to take into account not only the conjugacy class of a lattice group in $\Gamma_{3,1}$ as was done in $\S 4$, but also the details of its inclusion relations and conjugacy class within any larger lattice group. The conjugacy classes in $\Lambda(K)$ determine all the 'essentially distinct' variant structures that can result from symmetry-breaking distortions of a 2-lattice with metric $K$ while remaining in a $\mathcal{N}(K)$; in this way one can have a complete description of the distinct 'paths' for symmetry loss that exist for a crystal in the configuration $K$. This also allows us to describe the structure of the neighbourhood $\mathcal{N}(K)$. Again from the Propositions above, we have that $\mathcal{N}(K)$, which always contains a non-empty portion of the fixed set $I(\Lambda(K))$, for instance $K$ itself, contains a non-empty portion of any fixed set $I\left(\Lambda^{\prime}\right)$ if and only if $\Lambda^{\prime}$ is a subgroup of $\Lambda(K)$. Consequently, when all the lattice (sub)groups and the conjugacy classes in $\Lambda(K)$ are known, one only needs to determine the corresponding fixed sets through relations (16)-(18), (20)-(21). These describe completely the structure of an E-P neighbourhood of $K$, when the elements of $\mathcal{Q}_{3,1}^{e s s}$ that are within a small enough distance from $K$ are considered.

In the final Sections we give examples illustrating this procedure in two physically interesting cases: the neighbourhoods of the diamond structure (metrics of type 29) and the neighbourhoods of the hexagonal close-packed structure (metrics of type 27). Phases of C, Si, Sn ( $\alpha$-tin), for instance, crystallise in diamond-like structures; the h.c.p. structure is found in many metals, such as Ti, Co, Zr, Zn. The analysis presented here can actually be applied to determine the structure of any E-P neighbourhoods in $\mathcal{Q}_{3,1}^{\text {ess }}$ (or $\left.\mathcal{D}_{3,1}^{e s s}\right)$, and also for more complex multilattices.

\subsection{Notation}

Given a set of descriptors $\left(\mathbf{e}_{1}, \mathbf{e}_{2}, \mathbf{e}_{3}, \mathbf{p}\right) \in \mathcal{Q}_{3,1}^{\text {ess }}$, we shall use the following shorthand notation for the symmetry matrices $\mu$ belonging to its lattice group (see the definition in (10); see also [10]):

$$
\mu=\left(\text { symmetry operation }\left|l^{1} l^{2} l^{3}\right| \alpha\right)
$$

where:

- In the 'symmetry operations', a rotation is denoted by its period: the exponent ' + ' or ' - ' indicates whether the rotation is clock- or 
counterclock-wise, respectively, while the index indicates the rotation axis, for instance:

$$
4_{c}^{+} \text {is the four-fold rotation about an axis } c
$$

(this follows, in part, the notation in the International Tables for Crystallography [17]). The rotation axis is given as a combination of three directions $a, b, c$, which are the conventional axes of the lattice cell. ${ }^{11}$

- The matrix -1 (central symmetry) is indicated by $\overline{1}$, as is customary in crystallographic usage; thus, in general, an overbar indicates the opposite of a given operation (notice that $\overline{2}$ is a mirror symmetry).

- The triple of integers $\left(l^{1} l^{2} l^{3}\right)$ and the scalar $\alpha= \pm 1$ are those in formula (10).

The notation used for the lattice groups of the variants contained in an E-P neighbourhood is meant to recall the symmetry properties of the corresponding 2- lattices, as follows:

$$
\Lambda_{\text {type }}^{\text {axes }}
$$

here, 'type' refers to the arithmetic type number given in Table 1 or 2; 'axes' refers to a characteristic symmetry axis (or set of axes) in the centre lattice of the neighbourhood, and that remain also in the lower-symmetry variant after the distortion with loss of symmetry takes place. For instance, $\Lambda_{22}^{b}$ indicates the lattice group of a monoatomic 2-lattice of type 22 (whose Bravais type is centred tetragonal) with a four-fold axis along a direction $b ; \Lambda_{17}^{a, b, c}$ is the lattice group of a (face-centred orthorhombic) monoatomic 2-lattice of type 17 , with three mutually perpendicular two-fold axes along the directions $a, b$, and $c$.

For brevity, instead of listing the group elements we often indicate only the generators of the lattice groups:

$$
\Lambda=\langle\text { generator } 1 \text {, generator } 2 \text {, generator } 3, \ldots\rangle \text {; }
$$

\footnotetext{
${ }^{11}$ As we will only treat explicitly the cases of a cubic and of an hexagonal centre, we will only consider two choices of the axes $a, b, c$; in the cubic case they are along the three four-fold axes; in the hexagonal case they are along a typical hexagonal basis, with $a$ and $b$ along hexagon sides on the basal plane and 120 degrees apart, and $c$ perpendicular thereto (parallel to the six-fold axis). Recall, however, that in the matrix $\mu$ there actually appears, by definition, the 3 by 3 matrix with integral entries representing the symmetry operation with respect to the selected basis $\left(\mathbf{e}_{1}, \mathbf{e}_{2}, \mathbf{e}_{3}\right)$, which, in general, does not coincide with the conventional basis giving the axes $a, b, c$. The conventional cells for the 14 Bravais types are given in the International Tables; they are unit cells for the primitive types, but, for the centred types, they are non-unit cells that exhibit more clearly the lattice symmetry. For instance, the directions $a, b, c$ above are unit-cell axes only for the hexagonal case.
} 
we never indicate the generator $\overline{1}$, which, as recalled earlier on, is always present in the lattice groups of monoatomic 2-lattices, as they are all centrosymmetric.

\section{First example: neighbourhoods of a diamond metric (type 29) - Figure 2}

Let us consider an element $K_{29}$ of type 29 in $\mathcal{Q}_{3,1}^{\text {ess }}$ : this is the metric of a monoatomic 2-lattice with the diamond structure; for instance, we take $K_{29}$ to coincide with the metric given for type 29 in Table 1, whose lattice group we denote by $\Lambda_{29}^{a, b, c}$ (generators for it are given in the Table). This group is isomorphic to the cubic holohedry $4 / m \overline{3} 2 / m$, and has 48 elements; it is characterized by three four-fold operations with axes $a, b, c$ positioned as mentioned in the preceding footnote.

In this Section we describe the structure of the E-P neighbourhoods in $\mathcal{Q}_{3,1}^{\text {ess }}$ of the diamond metric $K_{29}$. As explained earlier, to this end we must

find all the subgroups of $\Lambda_{29}^{a, b, c}$ that are themselves lattice groups; then, the metrics in the neighbourhood of $K_{29}$ are those belonging to the the fixed sets of such lattice (sub)groups, and close enough to $K_{29}$. For brevity, in what follows we focus on the subgroups and their conjugacy classes, which give the essential information; their fixed sets then can be obtained routinely by using the conjugation matrices and formulas (16)-(18), (20)-(21), together with the data on 2-lattice metrics contained in Table 1.

The lattice (sub)groups of $\Lambda_{29}^{a, b, c}$ are not difficult to list, based on the results on 1-lattices given in $[29,26]$. Indeed, we mentioned earlier that, as for 1-lattices, also the monoatomic 2-lattice types, except for type 25, all have holohedral lattice groups. As no trigonal lattice group of type 25 is contained in $\Lambda_{29}^{a, b, c}$, its lattice (sub)groups must all be holohedral; therefore they reproduce the same group-subgroup diagram as for the cubic lattice groups of 1-lattices examined in [29, 26]. Fig. 2 represents this diagram for $\Lambda_{29}^{a, b, c}$, the details of which are described in the rest of this Section.

\subsection{Tetragonal subgroups (type 22) - tin pest}

Within $\Lambda_{29}^{a, b, c}$ there exist three (centred) tetragonal lattice (sub)groups, isomorphic to the holohedry $4 / m 2 / m 2 / m$, with 16 elements; they are all conjugate in $\Lambda_{29}^{a, b, c}$ - and thus, a fortiori, in $\Gamma_{3,1}$ (they are all of type 22 ). Explicitly, the three tetragonal subgroups are:

$$
\begin{aligned}
& \Lambda_{22}^{b}=\left(4_{a}^{-}|100| \overline{1}\right) \cdot \Lambda_{22}^{c} \cdot\left(4_{a}^{-}|100| \overline{1}\right)^{-1} \\
& \Lambda_{22}^{a}=\left(4_{b}^{+}|100| \overline{1}\right) \cdot \Lambda_{22}^{c} \cdot\left(4_{b}^{+}|100| \overline{1}\right)^{-1}
\end{aligned}
$$


where

$$
\Lambda_{22}^{c}=\left\langle\left(4_{c}^{+}|010| \overline{1}\right),\left(2_{a}|0 \overline{1} 0| 1\right)\right\rangle
$$

is conjugated to the group given in Table 1 (for clarity, in these formulas and in the following analogous ones, we indicate the group operation, that is, matrix multiplication, by a ' ''). Each one of the above lattice groups is characterised by a four-fold operation with axis along one of the axes $a$, $b$, or $c$, as indicated in their symbols. The symmetry-breaking distortion that produces, from the original diamond 2-lattice of type 29, a tetragonal 2-lattice of type 22, can be determined from the metric matrices and shift vectors indicated explicitly in Table 1 . In this case the deformation is any stretch along a cubic axis, with no extra degrees of freedom for the shift vector, as the latter is confined, in type 22 as well as in type 29, to a specific position in the periodicity cell. This distortion produces a 2-lattice with a metric of type 22 (see Table 1 ) which has $48 \div 16=3$ variants when its $\Lambda_{29}^{a, b, c}$-orbit is considered as in formula (20); each variant metric has one of the three lattice groups above.

Tin (Sn) is a material that undergoes a phase transformation between a diamond-like cubic structure to a set of three tetragonal variants as described here. The high-symmetry cubic phase is $\alpha$ - (or gray) tin, stable under about $285 \mathrm{~K}$, the low-symmetry product phase is $\beta$ - (or white) tin, stable above this temperature. The $\alpha-\beta$ transition in Sn (which actually takes places well below $285 \mathrm{~K}$ ) causes the powdering or flaking of tin exposed to low temperatures; this was called 'tin pest' in earlier times, as it badly damaged the organ pipes or roof tiles of North-European churches during cold winters (it seems it also disintegrated the buttons of the uniforms of Napoleon's soldiers in Russia). An E-P neighbourhood of $K_{29}$ as considered here is the natural domain for a $\Lambda_{29}^{a, b, c}$-invariant, temperature-dependent energy function for the $\alpha-\beta$ transition in tin; its absolute minimizers would be at the centre of the neighbourhood (metric $K_{29}$ ) for lower temperatures, and on the three tetragonal variants mentioned above for higher temperatures, with a suitable subcritical (to account for hysteresis) bifurcation connecting these extremal branches (see also footnote 12 on a possible further tin phase).

\subsection{Rhombohedral subgroups (type 24)}

Within $\Lambda_{29}^{a, b, c}$ there is also a (unique) conjugacy class constituted of four rhombohedral lattice groups (type 24, 12 elements each), given by:

$$
\Lambda_{24}^{a+b+c}=\left\langle\left(3_{a+b+c}^{+}|000| 1\right),\left(2_{a-b}^{+}|000| \overline{1}\right)\right\rangle,
$$


and

$$
\begin{aligned}
& \Lambda_{24}^{a-b-c}=\left(2_{a}^{+}|0 \overline{1} 0| 1\right) \cdot \Lambda_{24}^{a+b+c} \cdot\left(2_{a}^{+}|0 \overline{1} 0| 1\right)^{-1}, \\
& \Lambda_{24}^{a-b+c}=\left(2_{b}^{+}|00 \overline{1}| 1\right) \cdot \Lambda_{24}^{a+b+c} \cdot\left(2_{a}^{+}|00 \overline{1}| 1\right)^{-1}, \\
& \Lambda_{24}^{a+b-c}=\left(2_{c}^{+}|\overline{1} 00| 1\right) \cdot \Lambda_{24}^{a+b+c} \cdot\left(2_{c}^{+}|\overline{1} 00| 1\right)^{-1} .
\end{aligned}
$$

These lattice groups are isomorphic to the holohedry $\overline{3} 2 / m$; their ternary axes are, as indicated by their symbols, along the main diagonals of the cubic cell in the diamond structure. The distortions giving these rhombohedral groups are stretches of the diamond structure along such diagonals, with an extra degree of freedom for the shift vector, which in these variants is only restricted to be parallel to the ternary axis. In this case there exist $48 \div 12=4$ variants, each one of which has the lattice group above with the corresponding ternary axis. Their metrics can be obtained in the usual way from the metric and shift vector indicated for type 24 in Table 1, together with the conjugation matrices above used in formulas (18) and (21).

\subsection{Orthorhombic subgroups (types 17 and 18)}

There are two distinct conjugacy classes of orthorhombic subgroups in the lattice group $\Lambda_{29}^{a, b, c}$; correspondingly, there are two orthorhombic variant structures in the E-P neighbourhood of the diamond metric $K_{29}$ above. ${ }^{12}$ This means that, as for 1-lattices, there are two essentially distinct cubic-toorthorhombic symmetry-breaking mechanisms in this neighbourhood.

\subsubsection{Orthorhombic subgroup of type 17}

The first class is constituted by a single (face-centred orthorhombic) group of type 17, with 8 elements and three binary axes coinciding with the fourfold axes along the edges of the diamond cubic cell. This group is normal in $\Lambda_{29}^{a, b, c}$, and is given by

$$
\Lambda_{17}^{a, b, c}=\left\langle\left(2_{c}^{+}|\overline{1} 00| 1\right),\left(2_{a}^{+}|0 \overline{1} 0| 1\right)\right\rangle .
$$

One obtains a 2-lattice with this lattice group by stretching anisotropically the diamond structure along the edges of the cubic cell, and keeping the shift vector in the resulting position; this maintains a 'diamond-like' structure in the 2-lattice, whose skeleton, from cubic, becomes orthorhombic. There are $48 \div 8=6$ variants in this case, all of which share the same lattice group $\Lambda_{17}^{a, b, c}$ above.

\footnotetext{
${ }^{12}$ There also exists an orthorhombic phase of Sn, stable above about $160^{\circ} \mathrm{C}$, which is not well investigated; this form of tin perhaps crystallises in a (monoatomic) 2-lattice of type 17 or 18 as discussed here.
} 


\subsubsection{Orthorhombic subgroups of type 18}

The other conjugacy class of orthorhombic lattice (sub)groups of $\Lambda_{29}^{a, b, c}$ is constituted by three (body-centred orthorhombic) groups of type 18; the three mutually perpendicular 2-fold axes characterising each group are along the two diagonals of a face in the cubic diamond cell and along the cubic edge perpendicular thereto. Explicilty, these groups are:

$$
\Lambda_{18}^{a \pm b, c}=\left\langle\left(2_{c}^{+}|\overline{1} 00| 1\right),\left(2_{a+b}^{+}|100| \overline{1}\right)\right\rangle,
$$

and

$$
\begin{aligned}
\Lambda_{18}^{a \pm c, b} & =\left(3_{a+b+c}^{-}|000| 1\right) \cdot \Lambda_{18}^{a \pm b, c} \cdot\left(3_{a+b+c}^{-}|000| 1\right)^{-1}, \\
\Lambda_{18}^{b \pm c, a} & =\left(3_{a+b+c}^{+}|000| 1\right) \cdot \Lambda_{18}^{a \pm b, c} \cdot\left(3_{a+b+c}^{+}|000| 1\right)^{-1} .
\end{aligned}
$$

The lattice distortion that produces these orthorhombic groups is an anisotropic strain along the three two-fold axes mentioned above; correspondingly, the shift vector can have any value of its component along the axis coming from the former cubic side. Again there are $48 \div 8=6$ variants of this type in the E-P neighbourhood of the diamond metric $K_{29}$, which pair-wise share the lattice groups above.

\subsection{Monoclinic subgroups (types 6 and 7)}

Analogously to the orthorhombic case, there exist in $\Lambda_{29}^{a, b, c}$ two conjugacy classes of monoclinic subgroups (all of which have 4 elements); their groups belong to the centred-monoclinic types 6 and 7, respectively.

\subsubsection{Monoclinic subgroups of type 7 (cubic edges)}

One of the monoclinic classes above is constituted by three monoclinic groups of type 7 , each of which has a two-fold axis whose direction is along a cubic edge (four-fold axis) in the diamond structure:

$$
\Lambda_{7}^{b}=\left\langle\left(2_{b}^{+}|00 \overline{1}| 1\right)\right\rangle,
$$

and

$$
\begin{aligned}
& \Lambda_{7}^{a}=\left(4_{c}^{-}|001| \overline{1}\right) \cdot \Lambda_{7}^{b} \cdot\left(4_{c}^{-}|001| \overline{1}\right)^{-1}, \\
& \Lambda_{7}^{c}=\left(4_{a}^{+}|001| \overline{1}\right) \cdot \Lambda_{7}^{b} \cdot\left(4_{a}^{+}|001| \overline{1}\right)^{-1} .
\end{aligned}
$$

There are $48 \div 4=12$ variants of this type in the E-P neighbourhoods of the diamond metric $K_{29}$; they share, in four-tuples, the above lattice groups. 


\subsubsection{Monoclinic subgroups of type 6 (cubic face-diagonals)}

The second monoclinic class is constituted by six lattice groups of type 6 , whose two-fold axes are along a the face-diagonals of the cubic cell in the diamond structure:

$$
\Lambda_{6}^{a-b}=\left\langle\left(2_{a-b}^{+}|000| \overline{1}\right)\right\rangle
$$

and

$$
\begin{aligned}
\Lambda_{6}^{a+b} & =\left(2_{b}^{+}|00 \overline{1}| 1\right) \cdot \Lambda_{6}^{a-b} \cdot\left(2_{b}^{+}|00 \overline{1}| 1\right)^{-1}, \\
\Lambda_{6}^{a-c} & =\left(2_{b-c}^{+}|000| \overline{1}\right) \cdot \Lambda_{6}^{a-b} \cdot\left(2_{b-c}^{+}|000| \overline{1}\right)^{-1} \\
\Lambda_{6}^{a+c} & =\left(2_{b+c}^{+}|010| \overline{1}\right) \cdot \Lambda_{6}^{a-b} \cdot\left(2_{b+c}^{+}|010| \overline{1}\right)^{-1} \\
\Lambda_{6}^{b-c} & =\left(2_{a-c}^{+}|000| \overline{1}\right) \cdot \Lambda_{6}^{a-b} \cdot\left(2_{a-c}^{+}|000| \overline{1}\right)^{-1} \\
\Lambda_{6}^{b+c} & =\left(2_{a+c}^{+}|001| \overline{1}\right) \cdot \Lambda_{6}^{a-b} \cdot\left(2_{a+c}^{+}|001| \overline{1}\right)^{-1} .
\end{aligned}
$$

Again there are $48 \div 4=12$ variants of this type in the neighbourhoods, which, in pairs, share the above six lattice groups.

\subsection{Triclinic subgroup (type 1)}

Finally, there is only (one class constitituted by) one triclinic subgroup in $\Lambda_{29}^{a, b, c}$ :

$$
\Lambda_{1}=\{1, \overline{1}\}
$$

Any triclinic metric, with lattice group $\Lambda_{1}$, has $48 \div 2=24$ variants in the E-P neighbourhoods of $K_{29}$.

\section{Second example: neighbourhoods of an h.c.p. metric (type 27) - Figure 3}

The h.c.p. structure is one of the most important for crystalline materials, and we analyse its E-P neighbourhoods. For definiteness, we take the h.c.p metric in $\mathcal{Q}_{3,1}^{\text {ess }}$ to be the one, say $H_{27}$, given for type 27 in Table 1; we call $\Lambda_{27}^{c}$ its lattice group, which is isomorphic to the hexagonal holohedry $6 / m 2 / m 2 / m$ and has order 24 (generators of $\Lambda_{27}^{c}$ are given in the Table). Here $a, b, c$ are the usual hexagonal axes as mentioned in footnote 11.

Also the lattice (sub)groups of the hexagonal group $\Lambda_{27}^{c}$ are not too difficult to list, based on the results on 1-lattices recalled earlier on. We already mentioned that all monoatomic 2-lattice types, except for type 25, 
have holohedral lattice groups. Therefore, except for any non-holohedral subgroups of type 25, all the lattice (sub)groups in $\Lambda_{27}^{c}$ are holohedral and must thus reproduce the hexagonal group-subgroup relations indicated in $[29,26]$ for 1-lattices. To the latter one must in fact add a (non-holohedral) trigonal subgroup of type 25 . The diagram of the group-subgroup relations for the lattice (sub)groups of $\Lambda_{27}^{c}$ is as shown in Fig. 3, to which we make some brief comments in the rest of this Section.

\subsection{Trigonal subgroup (type 25)}

There are two subgroups of $\Lambda_{27}^{c}$ that are isomorphic to the trigonal (or rhombohedral) holohedry $\overline{3} 2 / m$ (12 elements); these groups are both normal in $\Lambda_{27}^{c}$. One such group is not the lattice group of any monoatomic 2-lattice: therefore we no longer consider it here. The other normal trigonal subgroup, given by:

$$
\Lambda_{25}^{c}=\left\langle\left(3_{c}^{+}|\overline{1} \overline{1} 0| \overline{1}\right),\left(\overline{2}_{a}^{+}|000| \overline{1}\right)\right\rangle,
$$

is indeed a lattice group in $\Gamma_{3,1}$, belonging to the conjugacy class related to the arithmetic type number 25 . The monoatomic 2-lattices that admit this lattice group have hexagonal Bravais lattice, but trigonal space group $(P \overline{3} 2 / m 1$, see Table 1$)$. The lattice distortion producing the lattice group $\Lambda_{25}^{c}$ is one in which the skeletal lattice remains hexagonal and the shift vectors can be changed to have a generic coordinate along the six-fold axis in the h.c.p structure, which for this reason becomes a trigonal axis; due to this change only three two-fold axes persist of the original six existing in the h.c.p, structure. The variants in this case are 2, which share the same lattice group above.

\subsection{Orthorhombic subgroups (type 14)}

There are three orthorhombic subgroups in $\Lambda_{27}^{c}$, which are all conjugate (they have 8 elements):

$$
\Lambda_{14}^{a, a+2 b, c}=\left\langle\left(2_{c}^{+}|001| \overline{1}\right),\left(2_{a}^{+}|000| \overline{1}\right)\right\rangle,
$$

and

$$
\begin{aligned}
\Lambda_{14}^{a+b, b-a, c} & =\left(6_{c}^{+}|011| \overline{1}\right) \cdot \Lambda_{14}^{a, a+2 b, c} \cdot\left(6_{c}^{+}|011| \overline{1}\right)^{-1} \\
\Lambda_{14}^{b, 2 a+b, c} & =\left(3_{c}^{+}|\overline{1} \overline{1} 0| 1\right) \cdot \Lambda_{14}^{a, a+2 b, c} \cdot\left(3_{c}^{+}|\overline{1} \overline{1} 0| 1\right)^{-1}
\end{aligned}
$$

These lattice groups belong to (the base-centred orthorhombic) type 14 in Table 1. Each group admits three mutually orthogonal binary axes among all those belonging to the parent h.c.p. lattice, that is, one parallel to 
an hexagonal side (direction [100]), a second one to an hexagonal side-axis (direction [120]), and a third one parallel to the optic axis [001]. The variants in this structure are $24 \div 8=3$, each one of which has a distinct lattice group.

The monoatomic 2-lattices belonging to this arithmetic type have the structure A20 of $\alpha$-U; an analysis of these variants and their presence in the E-P neighbourhood of an h.c.p. structure was given by [12] (see also the observations about twinning in $\mathrm{U}$ by $[16])$.

\subsection{Monoclinic subgroups (types 2, 6 and 7)}

In the h.c.p. lattice group $\Lambda_{27}^{c}$ there are 14 two-fold operations, which have, in pairs, opposite sign. This means that there are seven monoclinic subgroups in $\Lambda_{27}^{c}$, which are all lattice groups in $\Gamma_{3,1}$. They belong to three distinct conjugacy classes in $\Lambda_{27}^{c}$.

\subsubsection{Monoclinic subgroups of type 6}

One of these classes is constituted by three (centred monoclinic) lattice groups of type 6 , whose binary axes are along the hexagonal sides of the original h.c.p structure:

$$
\Lambda_{6}^{a}=\left\langle\left(2_{a}^{+}|000| \overline{1}\right)\right\rangle,
$$

and

$$
\begin{aligned}
\Lambda_{6}^{a+b} & =\left(6_{c}^{+}|011| \overline{1}\right) \cdot \Lambda_{6}^{a} \cdot\left(6_{c}^{+}|011| \overline{1}\right)^{-1} \\
\Lambda_{6}^{b} & =\left(3_{c}^{+}|\overline{1} \overline{1} 0| 1\right) \cdot \Lambda_{6}^{a} \cdot\left(3_{c}^{+}|\overline{1} \overline{1} 0| 1\right)^{-1} .
\end{aligned}
$$

In this case there are $24 \div 4=6$ variants, which pair-wise share the above three symmetry groups.

\subsubsection{Monoclinic subgroups type 7}

The second class is constituted by three (centred monoclinic) lattice groups of type 7 :

$$
\Lambda_{7}^{a+2 b}=\left\langle\left(2_{a+2 b}^{+}|00 \overline{1}| 1\right)\right\rangle,
$$

and

$$
\begin{aligned}
\Lambda_{7}^{b-a} & =\left(6_{c}^{+}|011| \overline{1}\right) \cdot \Lambda_{7}^{a+2 b} \cdot\left(6_{c}^{+}|011| \overline{1}\right)^{-1}, \\
\Lambda_{7}^{2 a+b} & =\left(3_{c}^{+}|\overline{1} \overline{1} 0| 1\right) \cdot \Lambda_{7}^{a+2 b} \cdot\left(3_{c}^{+}|\overline{1} \overline{1} 0| 1\right)^{-1} .
\end{aligned}
$$

In this case the binary axes are the side-axes in the basal plane of the h.c.p. structure. The variants are again 6 , pair-wise sharing the lattice groups above. 


\subsubsection{Monoclinic subgroup of type 2}

The third class is constituted by a single (normal) subgroup with arithmetic type 2 (primitive monoclinic):

$$
\Lambda_{2}^{c}=\left\langle\left(2_{c}^{+}|001| \overline{1}\right)\right\rangle
$$

the binary axis here is the six-fold axis in the h.c.p structure. Again there are 6 variants in this structure, which all share the same lattice group.

\subsection{Triclinic subgroup (type 1)}

As for the cubic case, the unique triclinic lattice group $\Lambda_{1}=\{1, \overline{1}\}$ is contained in $\Lambda_{27}^{c}$; any triclinic metric in the E-P neighbourhood of the h.c.p. metric $H_{27}$ has 12 variants.

\section{References}

[1] S. A. Adeleke. On the classification of monoatomic crystal multilattices. 1999. Preprint.

[2] J. M. Ball and R. D. James. Proposed experimental test of a theory of fine microstructure and the two well problem. Philos. Trans. Roy. Soc. London Ser. A, 338:389-450, 1992.

[3] K. Bhattacharya, R. D. James, and P. Swart. A nonlinear dynamic model for twin relaxation with application to $\mathrm{Au} 47.5$ at \% Cd and other shape-memory materials. In M. Yoo and M. Wuttig, editors, Twinning in advanced materials, New York, 1993. Theoretical Materials Science.

[4] L. Boyer, E. Kaxiras, J. Feldman, J. Broughton, and M. Mehl. Phys. Rev. Lett., 67:715-718, 1991.

[5] P. Engel. Geometric crystallography. Kluwer Academic Publishers, Dordrecht, 1986.

[6] J. L. Ericksen. Nonlinear elasticity of diatomic crystals. Internat. J. Solids and Structures, 6:951-957, 1970.

[7] J. L. Ericksen. Some phase transitions in crystals. Arch. Rational Mech. Anal., 73:99-124, 1980.

[8] J. L. Ericksen. Weak martensitic transformations in Bravais lattices. Arch. Rational Mech. Anal., 107:23-36, 1989.

[9] J. L. Ericksen. Equilibrium theory for X-ray observations. Arch. Rational Mech. Anal., 139:181-200, 1997. 
[10] J. L. Ericksen. On nonessential descriptions of crystal multilattices. Math. Mech. Solids, 4:363-392, 1998.

[11] J. L. Ericksen. On groups occuring in the theory of crystal multilattices. Arch. Rational Mech. Anal., 148:145-178, 1999.

[12] J. L. Ericksen. Twinning analyses in the X-ray theory. 1999. To appear.

[13] J. L. Ericksen. Notes on the X-ray theory. J. Elasticity, 55:201-218, 2000.

[14] G. Fadda and G. Zanzotto. The arithmetic symmetry of monoatomic 2-nets. Acta Cryst. Sect. A, 56:36-48, 2000.

[15] G. Fadda and G. Zanzotto. The arithmetic symmetry of multilattices: another view on the 29 types of monoatomic 2-lattices. 2000. Preprint.

[16] F. C. Frank. A note on twinning in alpha-uranium. Acta Met., 1:71-74, 1953.

[17] T. Hahn, editor. International Tables for Crystallography, Volume A. Reidel Publishing Company, Dordrecht, Boston, 1996.

[18] R. D. James. The stability and metastability of quartz. In S. S. Antman, J. L. Ericksen, D. Kinderlehrer, and I. Müller, editors, Metastability and incompletely posed problems, volume 3 of IMA Volumes in Mathematics and its applications. Springer-Verlag, Berlin, New York, Heidelberg, 1987.

[19] R. D. James and K. F. Hane. Martensitic transformations and shape memory materials. Acta Materialia, 2000. To appear.

[20] L. D. Landau and E. M. Lifshitz. Statistical physics. Pergamon Press, Oxford, New York, 1959.

[21] L. Michel. Bravais classes, Voronoï cells, Delone symbols. In T. Lulek, W. Florek, and S. Walcerz, editors, Symmetry and structural properties of condensed matter, Singapore, 1995. Academic Press.

[22] G. P. Parry. On diatomic crystals. Internat. J. Solids and Structures, 14:283-287, 1978.

[23] M. Pitteri. Reconciliation of local and global symmetries of crystals. $J$. Elasticity, 14:175-190, 1984.

[24] M. Pitteri. On ( $\nu+1)$-lattices. J. Elasticity, 15:3-25, 1985.

[25] M. Pitteri and G. Zanzotto. Beyond space groups : the arithmetic symmetry of deformable multilattices. Acta Cryst. Sect. A, 54:359-373, 1998. 
[26] M. Pitteri and G. Zanzotto. Continuum models for phase transitions and twinning in crystals. Chapman and Hall, London, 2000. In preparation.

[27] S. Sternberg. Group theory and physics. Cambridge University Press, Cambridge, 1994.

[28] Akademische Verlagsgesellschaft, Leipzig, 1913-1940.

[29] G. Zanzotto. Weak and symmetry-breaking transitions in simple lattices. 1999. Preprint. 


\section{Captions to Tables}

\section{Table 1}

The 29 arithmetic types of monoatomic 2-lattices in three dimensions. The skeletal lattice metric is given for each Bravais type. Each type is determined by the indicated shift vector, whose co-ordinates are given in terms of the conventional axes used in the International Tables (1996). The generators with $\alpha=1$ of the lattice groups are listed, together with the space group symbols and any existing Strukturbericht.

\section{Table 2}

Representation of the cells of the 29 types of monoatomic 2-lattices in three dimensions. In the centred cases the conventional (non-unit) cells used in the International Tables are shown, rather than the unit cells with two atoms. Bold and thin dashed lines help locating the points of the two congruent 1-lattices constituting the 2-lattice. For types (4), (22), (23), two equivalent cell configurations are displayed, the last two based on the equivalence of the body-centred and face-centred tetragonal skeletal cells. For ease of representation, cells (16) and (23) only show the atoms on the 'front' faces, while in cells (17) and (29) 'diamond-like' atomic bonds are indicated (in these cells, only thin dashed lines are used for clarity). The Strukturberichte are indicated in Table 1. 
Figure 1. Hierarchy of the monoatomic 2-lattice arithmetic types.

Figure 2. Diamond centre.

Figure 3. Hexagonal close-packed centre

Table 1. The 29 arithmetic types of monoatomic 2-lattices in three dimensions. The skeletal lattice metric is given for each Bravais type. Each type is determined by the indicated shift vector, whose co-ordinates are given in terms of the conventional axes used in the International Tables (1996). The generators with $\alpha=1$ of the lattice groups are listed, together with the space group symbols and any existing Strukturbericht.

Table 2. Representation of the cells of the 29 types of monoatomic 2lattices in three dimensions. In the centred cases the conventional (non-unit) cells used in the International Tables are shown, rather than the unit cells with two atoms. Bold and thin dashed lines help locating the points of the two congruent 1-lattices constituting the 2-lattice. For types (4), (22), (23), two equivalent cell configurations are displayed, the last two based on the equivalence of the body-centred and face-centred tetragonal skeletal cells. For ease of representation, cells (16) and (23) only show the atoms on the 'front' faces, while in cells (17) and (29) 'diamond-like' atomic bonds are indicated (in these cells, only thin dashed lines are used for clarity). The Strukturberichte are indicated in Table 1. 


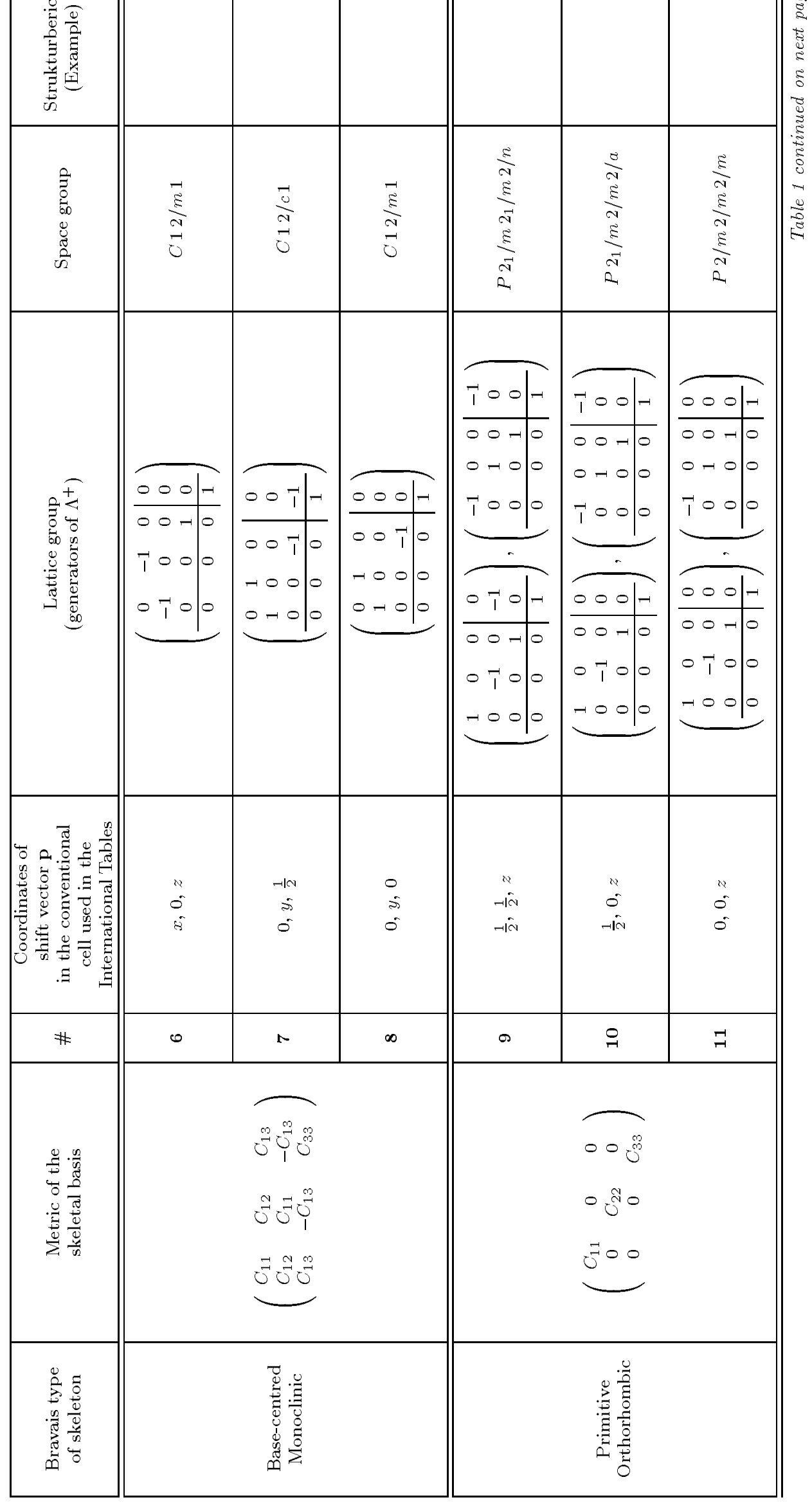




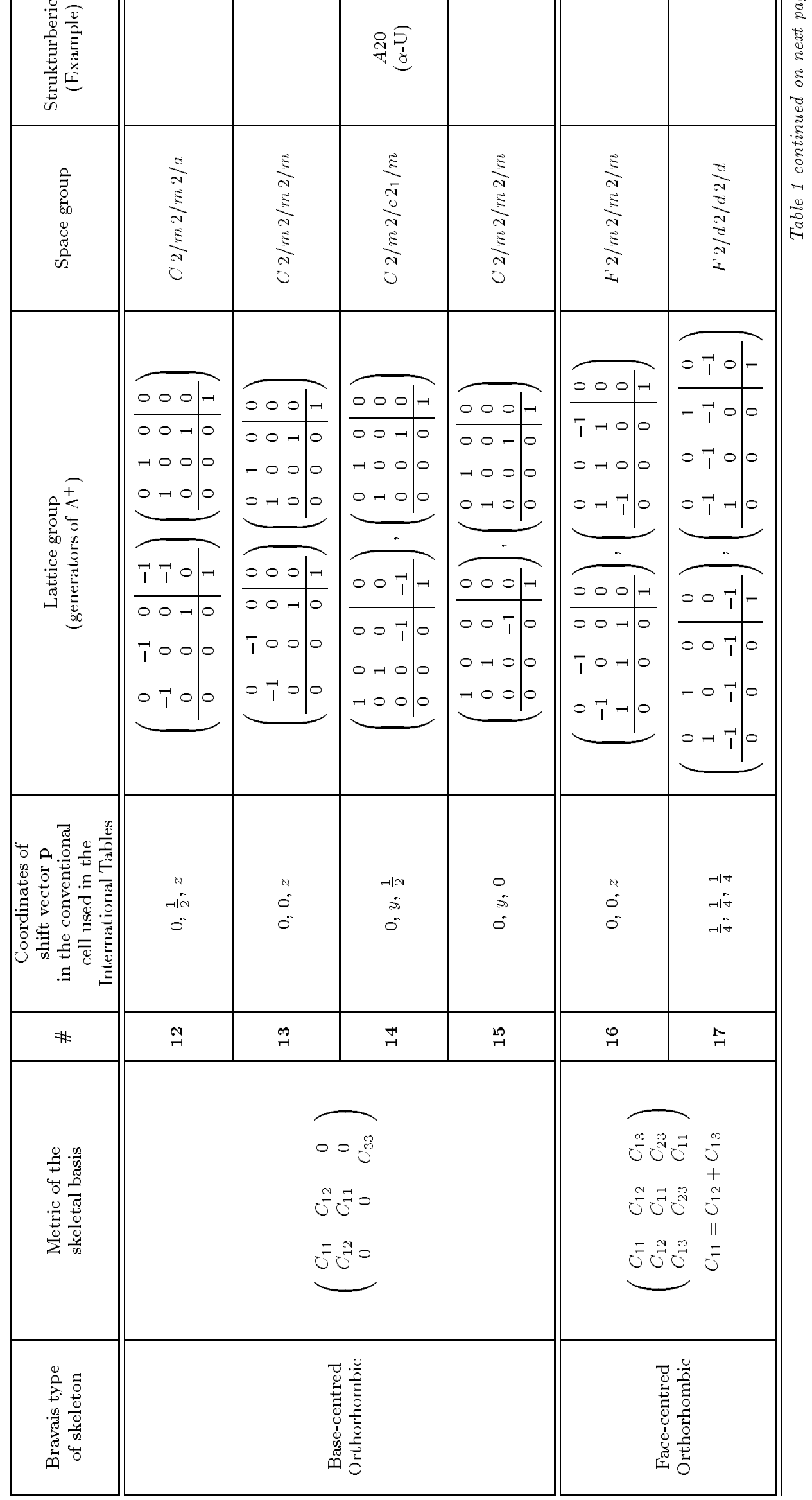




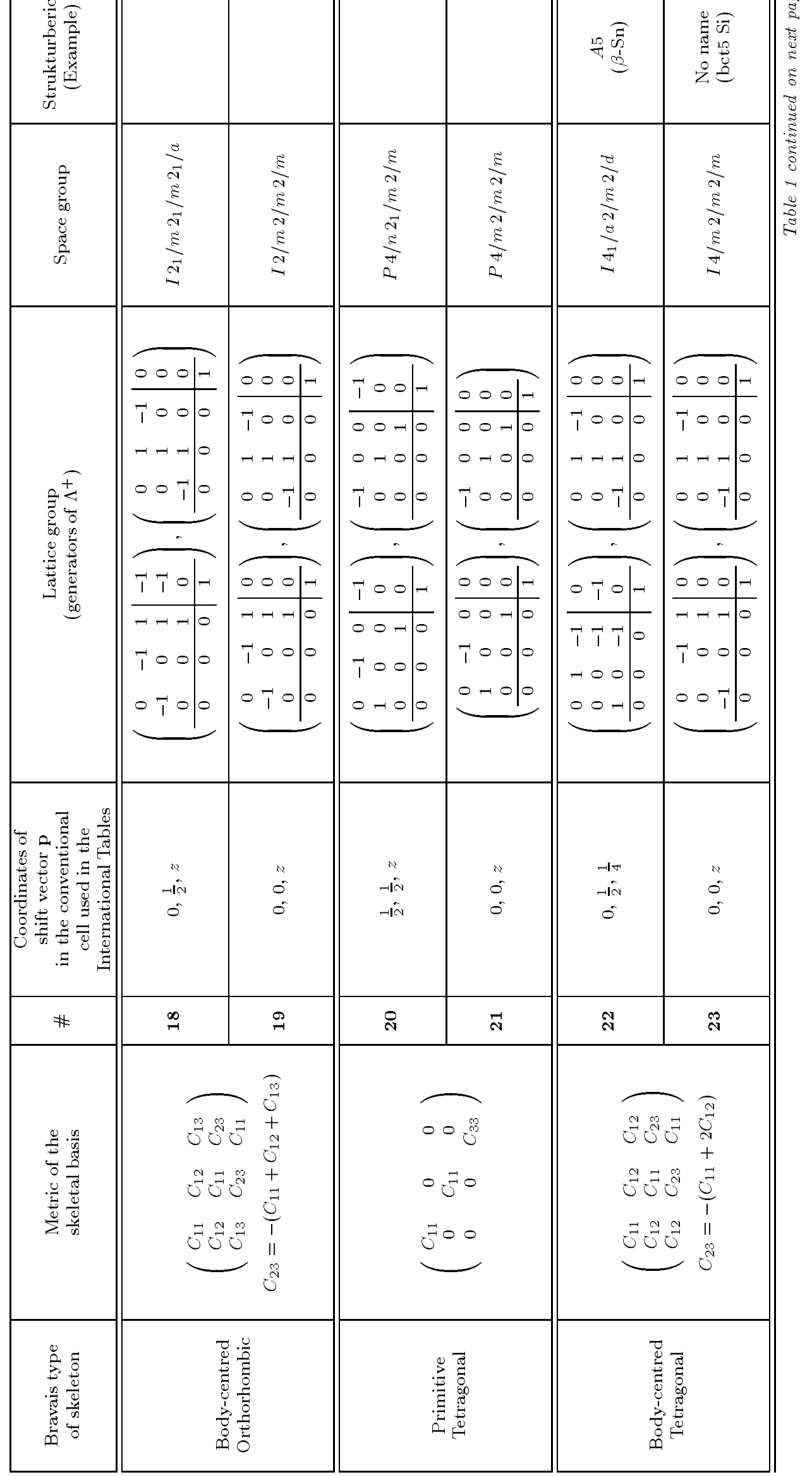




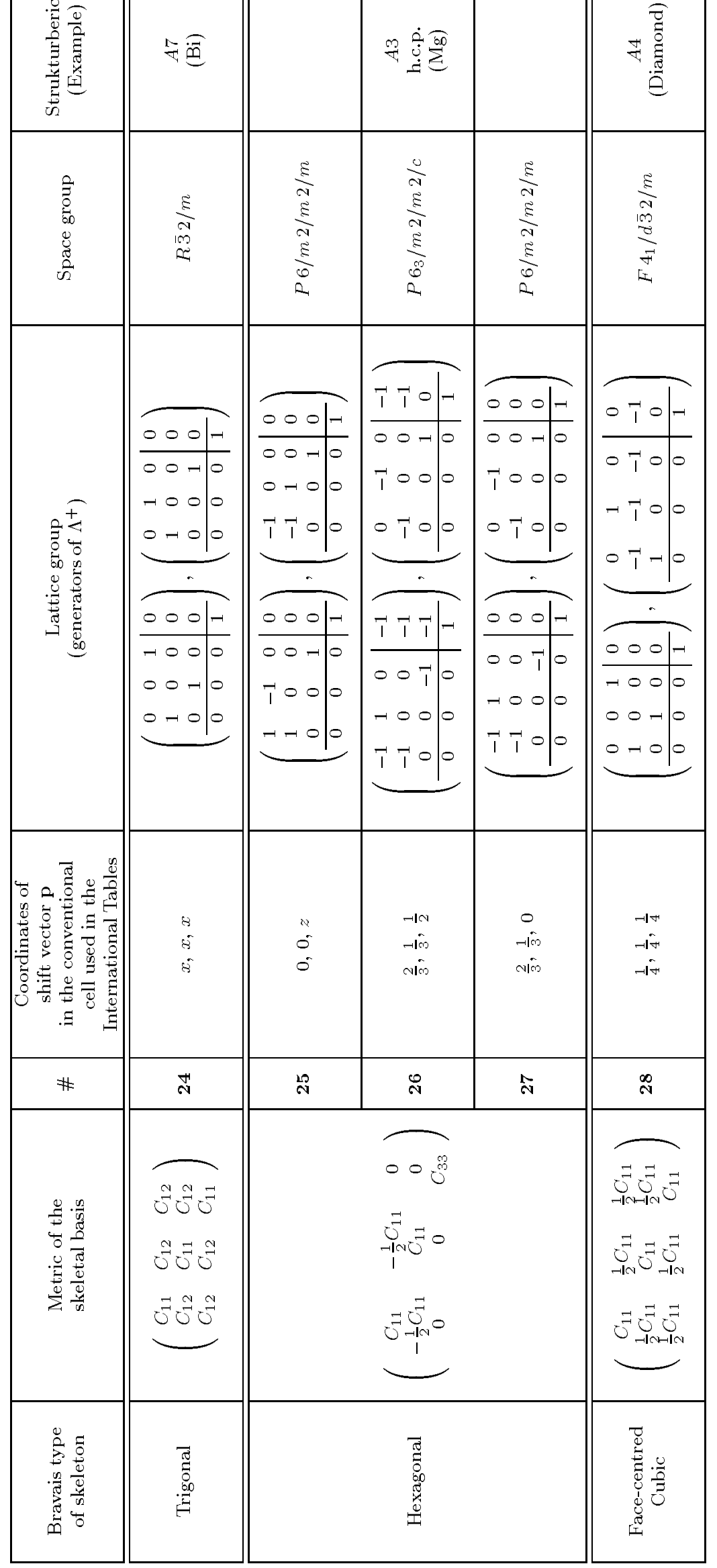


Table 2

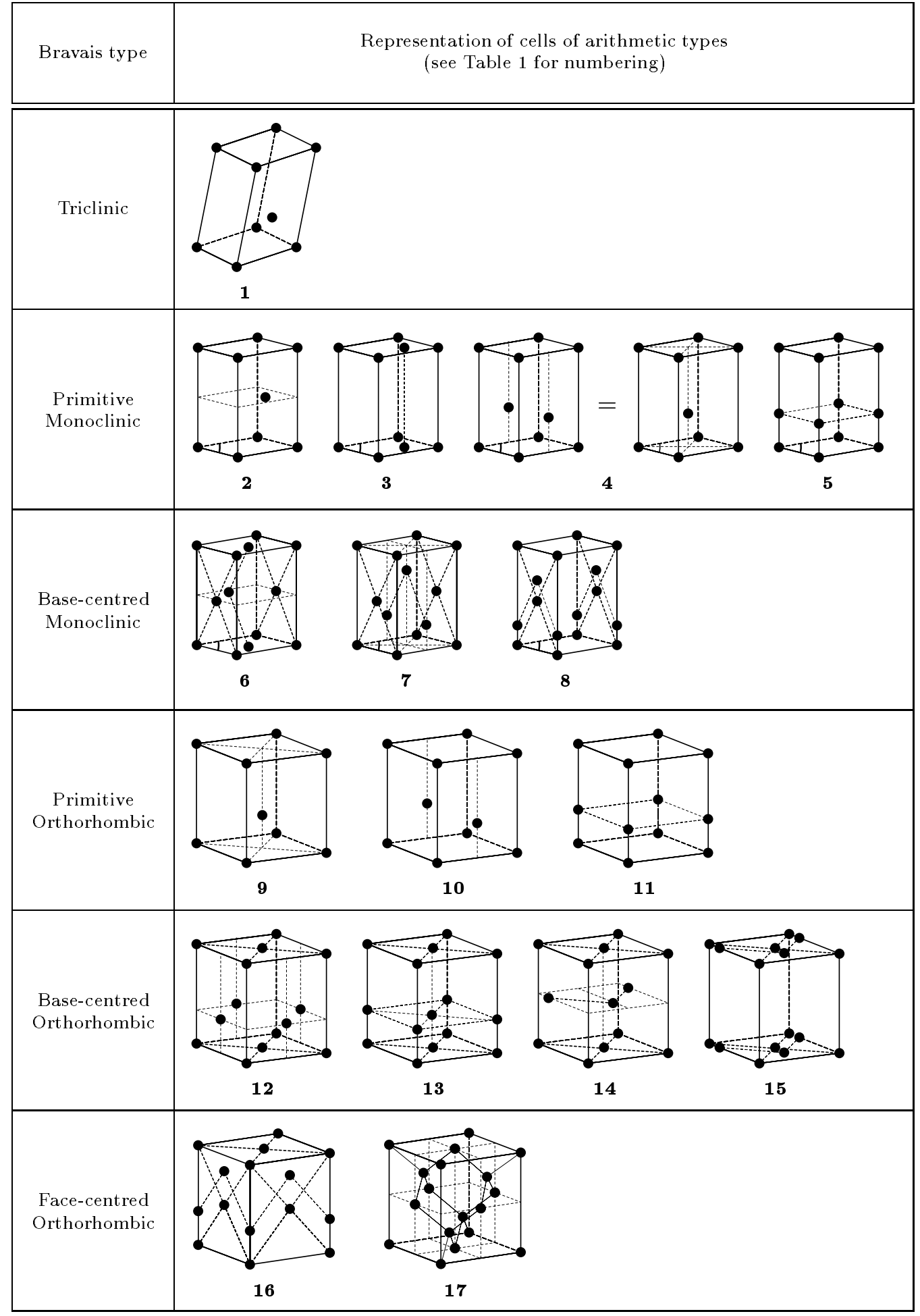

Table 2 continued on next page 


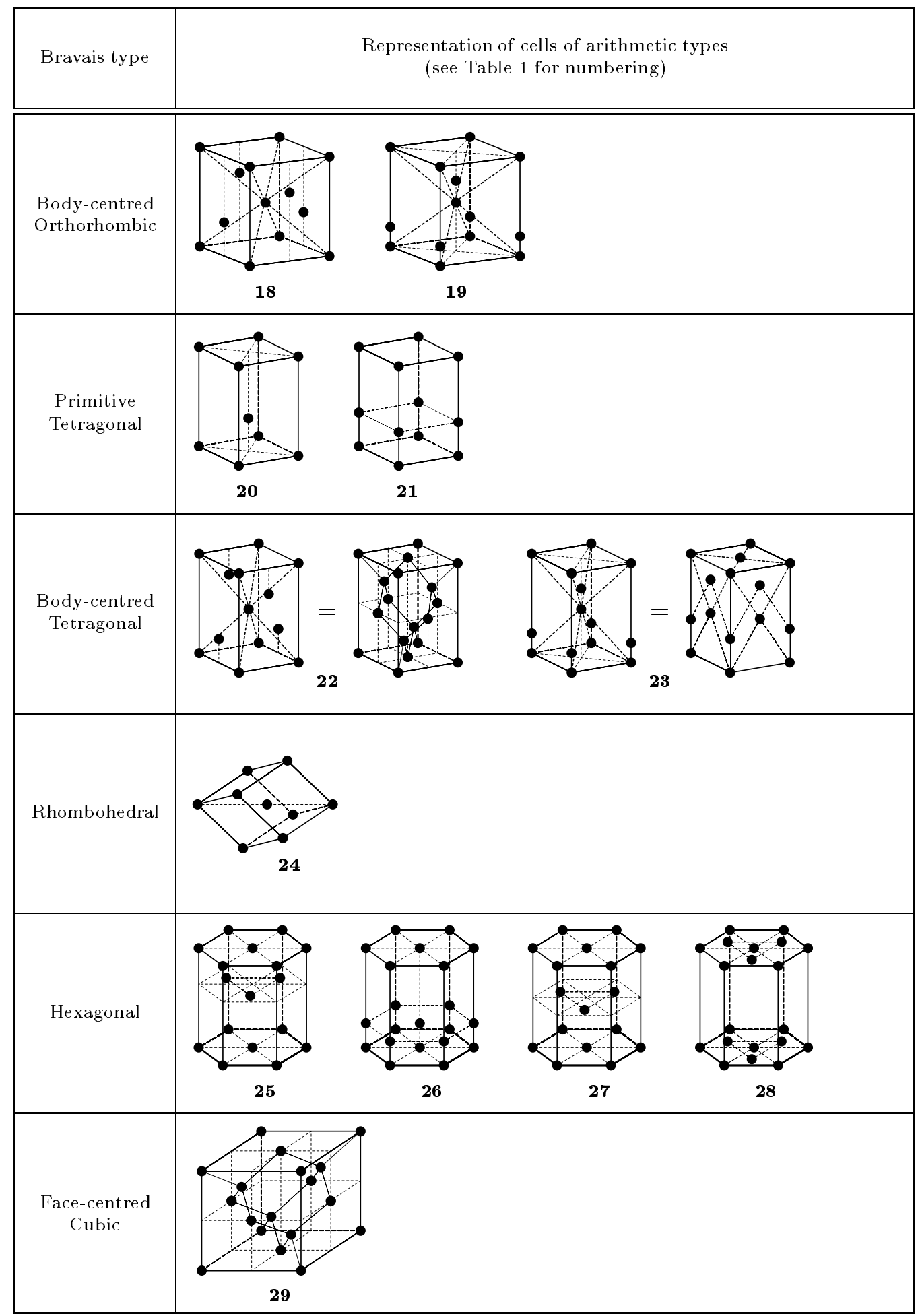




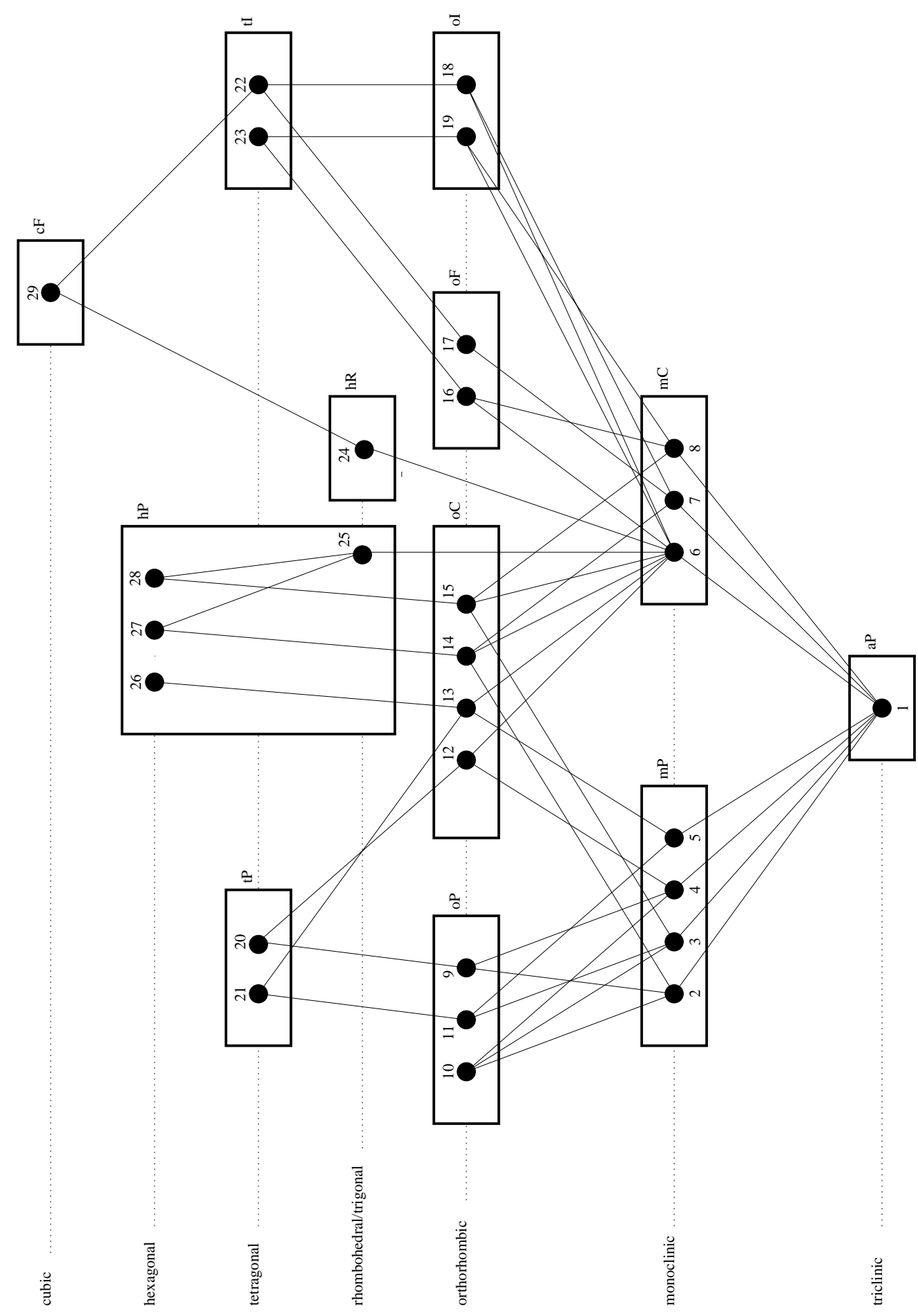

단)

$+\stackrel{0}{\circ}$

娄

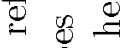

$\infty 0$

t5

ह

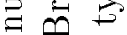

$\stackrel{9}{E}$

$\dot{0} \frac{0}{\pi}$

$\because \because$

政

ง

-

总息

है

००

\$

西西.

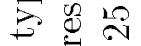

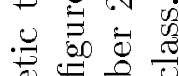

$\stackrel{\Xi}{\Xi} \Xi$

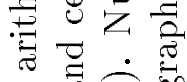

จ ㅇ 8

- 음

$=50$

¿ \&

क

๘气

ส.

$\because \pi$

․ 8

पह के

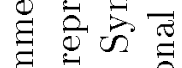

㞣

..

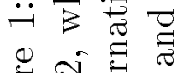

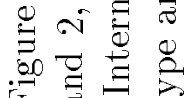




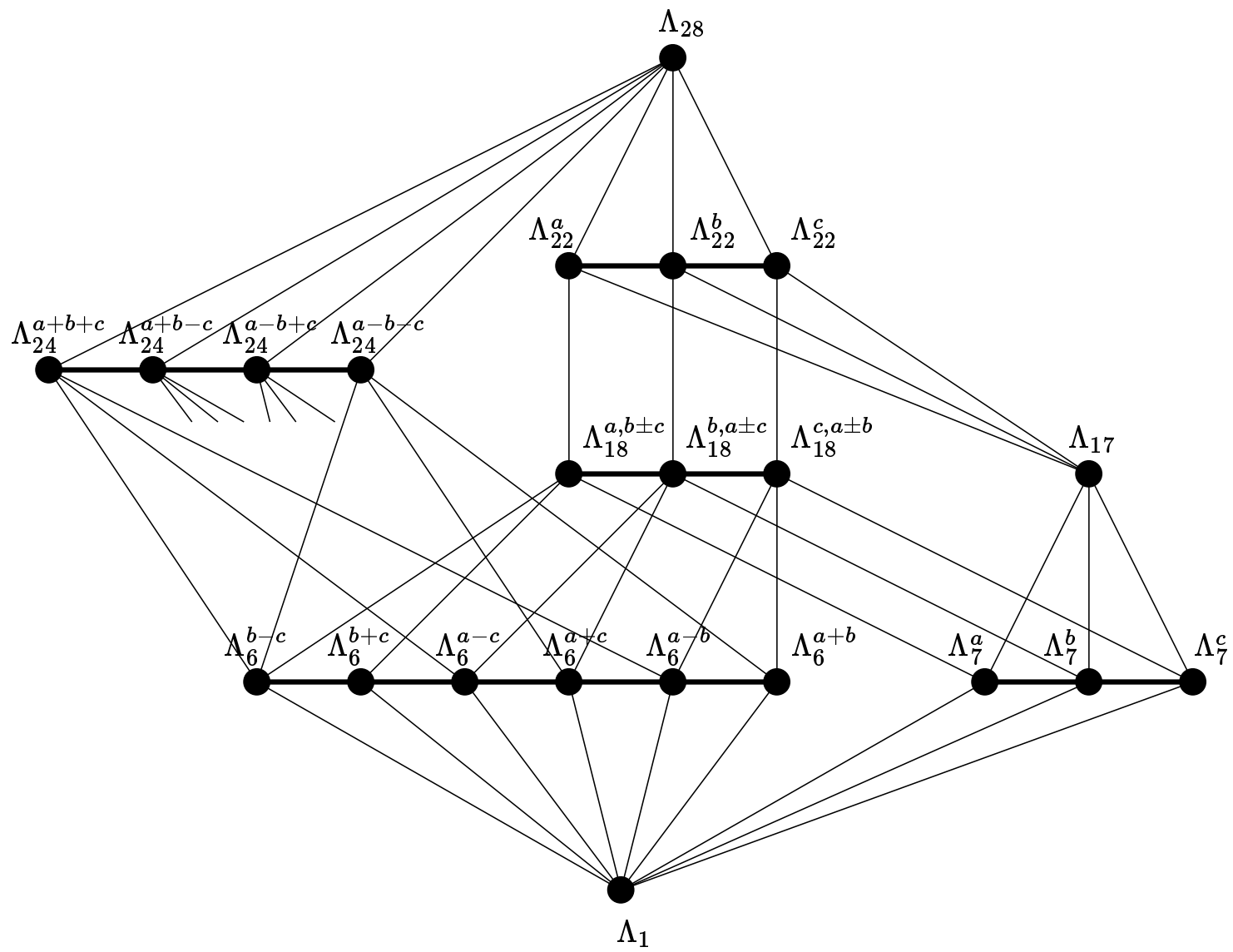

Figure 2: Diagram of the group-subgroup relations for the lattice (sub)groups of the diamond lattice group $\Lambda_{29}^{a, b, c}$ in Table 1. This is for the E-P neighbourhoods of a metric of type 29 (diamond structure). The thin slanted lines indicate inclusion (for clarity some lines are only partially indicated); the bold horizontal lines indicate the conjugacy classes in $\Lambda_{29}^{a, b, c}$. The symbols for the subgroups indicate their type number as in Tables 1 and 2 , and the direction of their main symmetry axes. See Section 6 for details. 


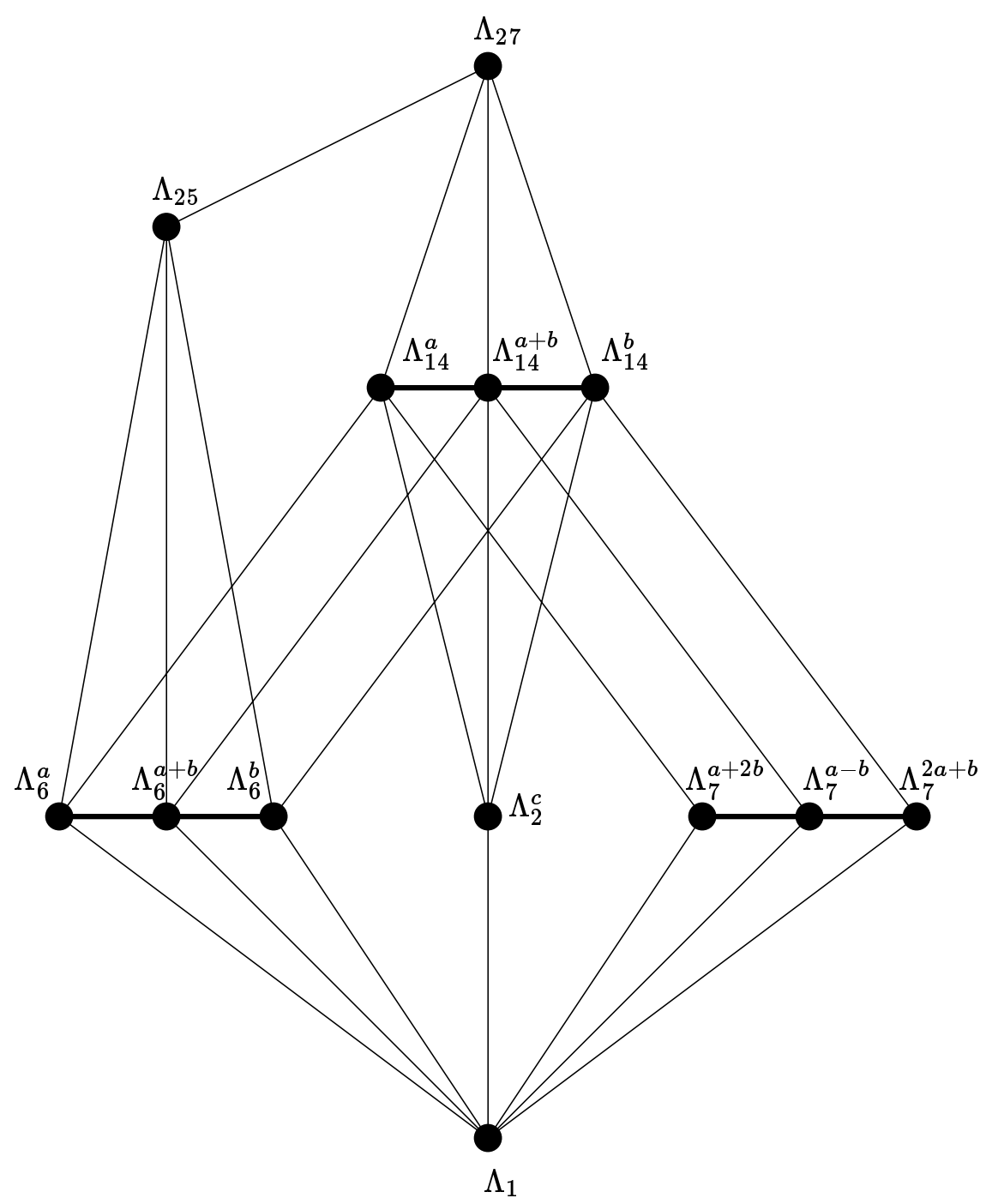

Figure 3: Diagram of the group-subgroup relations for the lattice (sub)groups of the h.c.p. lattice group $\Lambda_{27}^{c}$ in Table 1. This is for the E-P neighbourhoods of a metric of type 27 (hexagonal close-packed structure). The thin slanted lines indicate inclusion, the bold horizontal lines indicate the conjugacy classes in $\Lambda_{27}^{c}$. The symbols for the subgroups indicate their type number as in Tables 1 and 2, and the direction of their main symmetry axes. See Section 7 for details. 